\title{
Fermented Ginseng with Bifidobacterium Inhibits Angiogenesis of Human Umbilical Endothelial Cells in vitro and in vivo
}

\author{
Yu Jin Ko ${ }^{1}$, Seung Hee Park ${ }^{2}$, Byung Chul Park ${ }^{1}$, Yong-Hwa LeE ${ }^{2}$, and Jung-Ae Kim ${ }^{1}$,* \\ ${ }^{1}$ College of Pharmacy, Yeungnam University, Gyeongsan 712-749, Korea \\ ${ }^{2}$ R\&D Center, Jung San Biotechnology, Whasoung 445-964, Korea
}

(Received March 19, 2007; Accepted March 26, 2007)

\begin{abstract}
Ginseng is a widely-used alternative medicine for the treatment of cancer, diabetes, and cardiovascular diseases. Active components of $P$. ginseng, absorbed through gastrointestinal tract are the fermented ginsenosides by intestinal microorganisms. In the present study, we investigated the inhibitory effects of fermented ginseng with bifidobacterium (FGb) on the angiogenesis by analyzing in vitro tube formation and invasion assay using human umbilical vein endothelial cells (HUVECs), and in vivo angiogenesis using chick chorioallantoic membrane (CAM) assay. Treatment with FGb inhibited tube-like structure formation in a concentration-dependent manner. In addition, FGb significantly suppressed HUVEC invasion through Matrigel. Moreover, FGb dosedependently inhibited VEGF-induced angiogenesis in a CAM assay. These results suggest that FGb is a valuable anti-angiogenic remedy.
\end{abstract}

Key words $\square$ Fermenta ginseng, Angiogenesis, Tube formation, Migration, Chick chorioallantoic membrane

\section{INTRODUCTION}

Ginseng, the root of Panax ginseng C.A. Meyer, has been used in traditional medicine in Korea and East Asian countries for more than 1000 years. The effects of ginseng include general 'tonic', anti-fatigue, anti-stress, immunomodulatory, and anti-cancers (Shin et al, 2000; Yue et al., 2006). Nowadays, it is one of the world-wide alternative medicines for cancer, diabetes, and cardiovascular diseases. Up to now, more than 30 different ginsenosides have been isolated, each with a different set of properties. The ginsenosides can be subdivided as protopanaxadiol-containing compounds such as $\mathrm{Rg}_{3}$ and $\mathrm{Rh}_{2}$, and protopanaxatriol-containing ones such as $\mathrm{Rg}_{1}$ and $\mathrm{Rh}_{1}$. Among them, $\operatorname{Rg}_{1}$, the most abundant and active component, has been reported to promote angiogenesis in vitro and in vivo (Sengupta et al., 2004; Liang et al., 2005). In addition, $\operatorname{Rg}_{1}$ has been reported to induce vascular endothelial growth factor (VEGF) in human endothelial cells (Leung et al., 2006). In contrast, $\operatorname{Rg}_{3}$ has been reported to exhibit in vitro and in vivo anti-carcinogenic, antimetastatic and anti-angiogenic effects (Mochizuki et

*Corresponding author

Tel: +82-53-810-2816,

E-mail: jakim@yu.ac.kr al., 1995; Shinkai et al., 1996; Liu et al., 2000; Yun et al., 2001; Jin et al., 2006). In spite of various activities of ginsenosides, oral bioavailability of intact ginsenosides is extremely low since intact ginsenosides are hardly decomposed by gastric juice and poorly absorbed (Karikura et al., 1991; Tanizawa et al., 1993; Xu et al., 2003). However, protopanaxadiol-type ginsenosides such as $\mathrm{Rb}_{1}, \mathrm{Rb}_{2}$, and $\mathrm{Rc}$ are metabolized by colonic bacteria to 20-O-( $\beta$-D-glucopyranosyl)-20(S)-protopanaxadiol that is easily absorbed. In addition, it is known that an inhibitory effect on tumor cell invasion and angiogenesis is induced by the metabolite (Mochizuki et al., 1995). Furthermore, it has been demonstrated that the compound mediating in vivo antimetastatic effects of ginsenosides is the metabolite, $20-O-(\beta-\mathrm{D}-$ glucopyranosyl)-20(S)-protopanaxadiol (Hasegawa et al., 1997). It is now well recognized that the deglycosylation process of ginsenoside by colonic bacteria is crucial for its pharmacological expression.

Fermented Ginseng with Bifidobacterium (FGb) is obtained by fermenting ginseng power (sterilized at $121^{\circ} \mathrm{C}$ with pressure for $15 \mathrm{~min}$ ) with Bifidobacterium at $37^{\circ} \mathrm{C}$ for 7 days. Contrast to the intestinal bacteria, Bifidobacterium is beneficial to health and do not generate bad smell. In addition, FGb is known to contain 20-O-( $\beta$-D-glucopyranosyl)-20(S)-protopanaxadiol and 20(S)-protopanaxatriol, the biologically active compounds for 
anticancer effects (Korea patent 10-2004-0013653).

Angiogenesis is the physiological process in the body that involves the growth of new blood vessels from pre-existing vessels. In normal physiology, it is essential in reproduction, embryonic development, menstrual cycle and wound repair (Mousa et al., 2005; Rosenblatt and Azar, 2006). However, inappropriate angiogenesis is involved in the pathogenesis of many chronic diseases including tumor (Lin et al., 2006). The angiogenic process is composed of complex and diverse cellular actions such as extracellular matrix degradation, and proliferation, migration, and differentiation of endothelial cells, which is tightly regulated by angiogenic stimulators and inhibitors. Among the factors, VEGF is well known to play a key role in regulating normal and abnormal angiogenesis by stimulating endothelial cell migration and proliferation (Isner and Asahara, 1999). Tumor-induced angiogenesis is also driven by VEGF released by tumor cells in high metabolic demands, and is essential for the growth of tumor (Folkman, 1971; Blouw et al., 2003). Each of the angiogenesis elements, basement membrane disruption, cell migration, cell proliferation, and tube formation can be a target for angiogenesis intervention therapy, and thus for cancer therapy.

Although the ginseng colonic metabolite, 20-O- $(\beta-\mathrm{D}$-glucopyranosyl)-20(S)-protopanaxadiol, has been reported to inhibit tumor cell growth and anti-metastasis (Hasegawa et al., $1995 ; 1997 \mathrm{a})$, the anti-angiogenic effect of the metabolite, 20 $O$-( $\beta$-D-glucopyranosyl)-20(S)-protopanaxadiol, is not clearly reported yet. In the present study, we investigated the inhibitory effects of FGb on the VEGF-induced angiogenesis by analyzing in vitro tube formation and invasion assay using HUVECs, and in vivo angiogenesis using chick chorioallantoic membrane (CAM) assay.

\section{MATERIALS AND METHODS}

\section{Materials}

Human umbilical vein endothelial cells (HUVECs) were purchased from American Type Culture Collection (ATCC, Manassas, VA, U.S.A.). EGM-2 bullet kit which contains an endothelial cell basal medium-2 (EBM-2 medium) and EGM-2 singlequots (supplements and growth factors) were purchased from Cambrex (San Diego, CA, U.S.A). Glutamax and cortisone acetate were purchased from Gibco (Grand Island, NY, U.S.A.) and Sigma (St. Louis, MO, U.S.A.), respectively. Matrigel basement membrane matrix was purchased from BD Biosciences (San Diego, CA, U.S.A.). FGb was generously obtained from WonPharm Co., LTD (Iksan, Korea).

\section{Cell culture}

HUVECs were cultured in a gelatin-coated flask at $37^{\circ} \mathrm{C}, 5 \%$ $\mathrm{CO}_{2}$, with EBM-2 medium supplemented with $2 \%$ fetal bovine serum (FBS), ascorbic acid, hydrocortisone, human fibroblast growth factor (hFGF), vascular endothelial growth factor (VEGF), human epidermal growth factor (hEGF), long R insulin-like growth factor-1 ( $\mathrm{R}^{3}$-IGF-1), gentamicin sulfate (GA1000) and heparin. HUVECs were used between passages 2 and 6 for the whole experiment.

\section{Tube formation assay}

The tube formation assays were performed on 48-well plates coated with $100 \mu \mathrm{l}$ of matrigel basement membrane matrix per well and polymerized at $37^{\circ} \mathrm{C}$ for $30 \mathrm{~min}$. HUVECs were suspended in 2\% FBS and other supplement containing EBM-2 medium. Cells were plated on matrigel at a density of $5 \times 10^{4}$ cells per well, and test compound was added to the culture medium. After $14 \mathrm{~h}$, four fields were randomly selected from each culture and photographed with a CCD camera (TE2000U, Nikon, Japan).

\section{Cell invasion assay}

The upper and lower parts of transwell (Corning Costar, Cambridge, MA, U.S.A.) inserts were coated with $40 \mu \mathrm{l}$ of matrigel $(1.0 \mathrm{mg} / \mathrm{ml})$ and $40 \mu \mathrm{l}$ of type I collagen $(0.5 \mathrm{mg} / \mathrm{ml})$, respectively. The transwell inserts were put in FBS-free EBM2-filled 24-well plate. To the lower chamber, 5\% FBS was added. HUVECs $\left(5 \times 10^{4}\right.$ cells/chamber $)$ were plated on the matrigel-coated transwell, and then, treated with FGb for $24 \mathrm{~h}$. After $24 \mathrm{~h}$, the inserts were taken out and the inner surface of the insert was carefully wiped out using a cotton swab. The cells that had invaded to the lower surface of the membrane were fixed with methanol and stained with hematoxylin and eosin. Cells in four random fields of the insert were counted under a light microscope (TMS, Nikon, Japan).

\section{Chick chorioallantoic membrane (CAM) Assay}

In vivo neovascularization was examined by the method previously described by Mousa et al (2005). Briefly, 10-day-old embryos were purchased from Baek-ja (Cheongsong, Korea), a farm, and were incubated at $37^{\circ} \mathrm{C}$ with $55 \%$ relative humidity. A small hole was punctured in the shell concealing the air sac using a hypodermic needle. A second hole was punctured in the shell on the broadside of the egg directly over avascular portion 
of the embryonic membrane, as observed during candling. A false air sac was created beneath the second hole by the application of negative pressure to the first hole, which caused the chick chorioallantoic membrane (CAM) to separate from the shell. A window, approximately $1.0 \mathrm{~cm}^{2}$, was cut in the shell over the dropped CAM with the use of a small crafts grinding wheel (Dremel, Racine WI, U.S.A.). Sterile filter disks (Whatman \#1 filter paper, U.K.) were soaked in $3 \mathrm{mg} / \mathrm{ml}$ cortisone acetate in a solution of $95 \%$ ethanol and water and subsequently air dried under sterile conditions. VEGF was used to grow vessels on the CAMs of 10-day-old chick embryos. Sterile filter disks absorbed with VEGF (15 $\mathrm{ng} / \mathrm{ml})$ dissolved in PBS containing $0.1 \%$ BSA were placed on growing CAMs. Then, test compounds or control vehicle was added directly to CAMs topically. CAM tissue directly beneath the VEGF-saturated filter disks was resected from embryos treated 72 hours previously with compound or control. CAM disks were harvested for light microscopy (Leica, Germany). The number of vessel branch points contained in a circular region equal to the area of filter disk was counted for each section.

\section{RESULTS}

\section{Inhibitory effects of FGb on tube formation of HUVECs}

Tube formation is one of the important events of angiogene- sis. We first examined the effect of FGb on the ability of endothelial cells to form tubular structures. HUVECs that were plated on Matrigel formed a tubular network within $14 \mathrm{~h}$, as evidenced by morphological changes (Fig. 1). However, treatment with various concentrations of FGb inhibited formation of tube-like structures in a concentration-dependent manner.

\section{Inhibitory effects of FGb on invasion of HUVECs}

Since endothelial cell invasion is one of the important criteria for angiogenesis, the invasion of HUVECs through matrigel-coated transwell inserts was tested. The cells that were suspended in serum-free medium and plated on matrigel-coated chambers invaded through the matrigel toward an angiogenic factor (FBS)-containing lower chamber. FGb inhibited HUVEC invasion in a concentration-dependent manner, and $200 \mu \mathrm{g} / \mathrm{ml}$ of FGb almost completely blocked the invasion (Fig. 2).

\section{FGb inhibits VEGF-induced angiogenesis in vivo}

To study in vivo anti-angiogenic effect of FGb, CAM of chick embryo was selected as an angiogenesis model system. The vasculature of the CAM treated with VEGF $(15 \mathrm{ng} / \mathrm{ml})$ for 3 days was highly increased compared to vehicle-treated control group in a morphological and quantitation analyses. Treatment with FGb (10 and $100 \mu \mathrm{g} / \mathrm{CAM})$ applied onto the CAM of 10 day-old chick embryo for 3 days significantly decreased
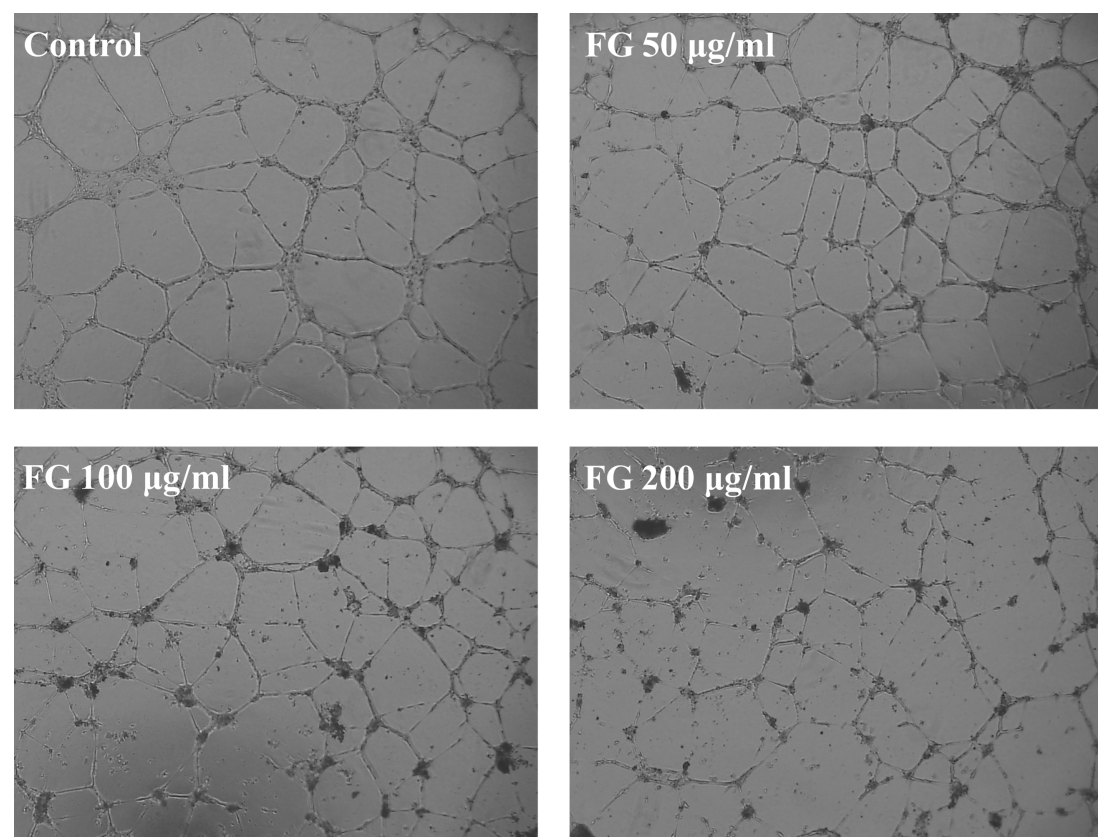

Fig. 1. Inhibitory effect of FGb on tube formation of endothelial cells. A 48-well plate was coated with $100 \mu 1$ of Matrigel basement membrane matrix per well and polymerized at $37^{\circ} \mathrm{C}$ for $30 \mathrm{~min}$. Cells were plated on top of Matrigel and treated with various concentrations of $\mathrm{FGb}$ for $14 \mathrm{~h}$ at $37^{\circ} \mathrm{C}$. These figures represent microscopic images of tube formation (magnification: $\times 40$ ). 

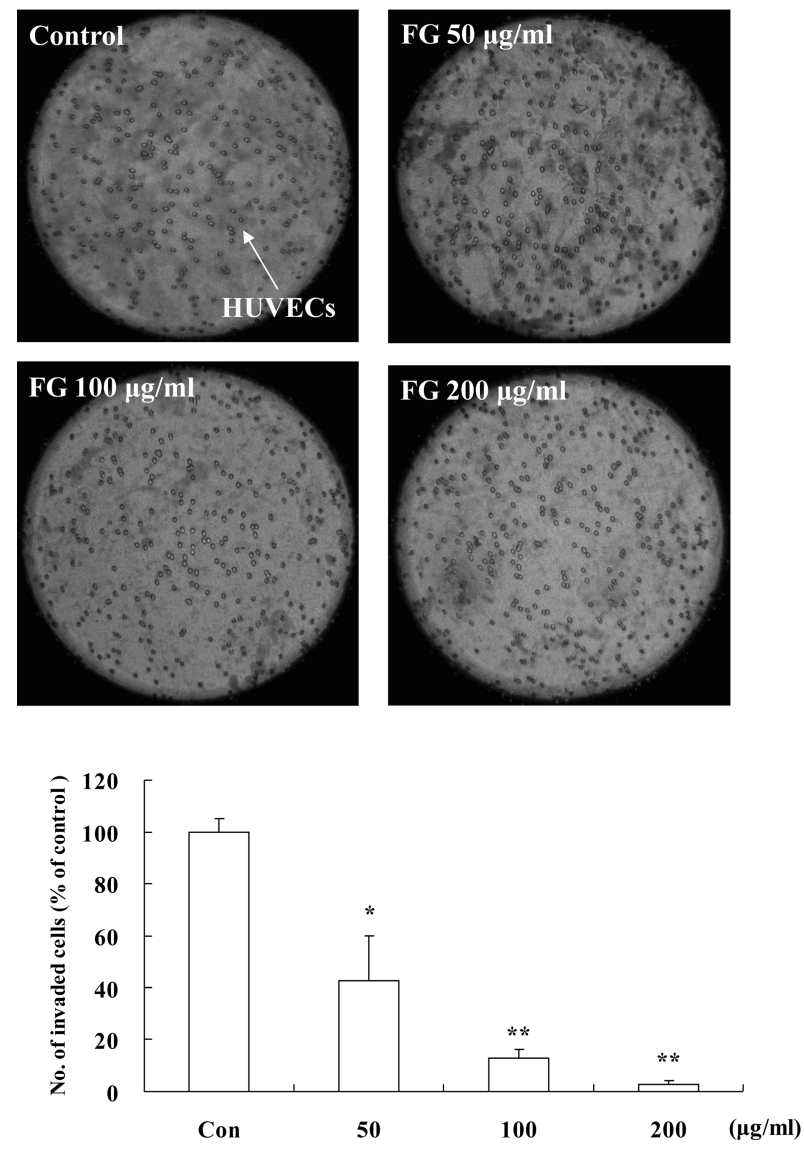

Fig. 2. Inhibitory effect of FGb on HUVEC invasion. The upper and lower parts of transwell chambers were coated with $40 \mu \mathrm{l}$ of Matrigel $(1.0 \mathrm{mg} / \mathrm{ml})$ and $40 \mu \mathrm{l}$ of type I collagen $(0.5 \mathrm{mg} /$ $\mathrm{ml})$, respectively. The medium in the lower chamber transwell contained 5\% FBS with supplement in EBM-2 medium. Cells suspended in serum- and supplement-free EBM-2 medium were plated on the Matrigel-coated inserts in the presence of various concentrations of $\mathrm{FGb}$ for $24 \mathrm{~h}$ at $37^{\circ} \mathrm{C}$. The data were the representative of three independent experiments showing microscopic images of invaded cells (magnification: $\times 200$ ). The bar graphs represent the relative number of invaded cells. $* P<$ 0.05 and $* * P<0.01$, compared to FGb-untreated control.

the VEGF-induced angiogenesis as shown in Fig. 3.

\section{DISCUSSION}

In the present study, we clearly demonstrated anti-angiogenic effects of FGb by analyzing in vitro tube formation and invasion assay using HUVECs, and in vivo angiogenesis using CAM assay.

Ginseng is frequently used as a crude substance and it is taken orally as a traditional medicine in Asian countries. Ginsenosides are among active components of ginseng, the root of
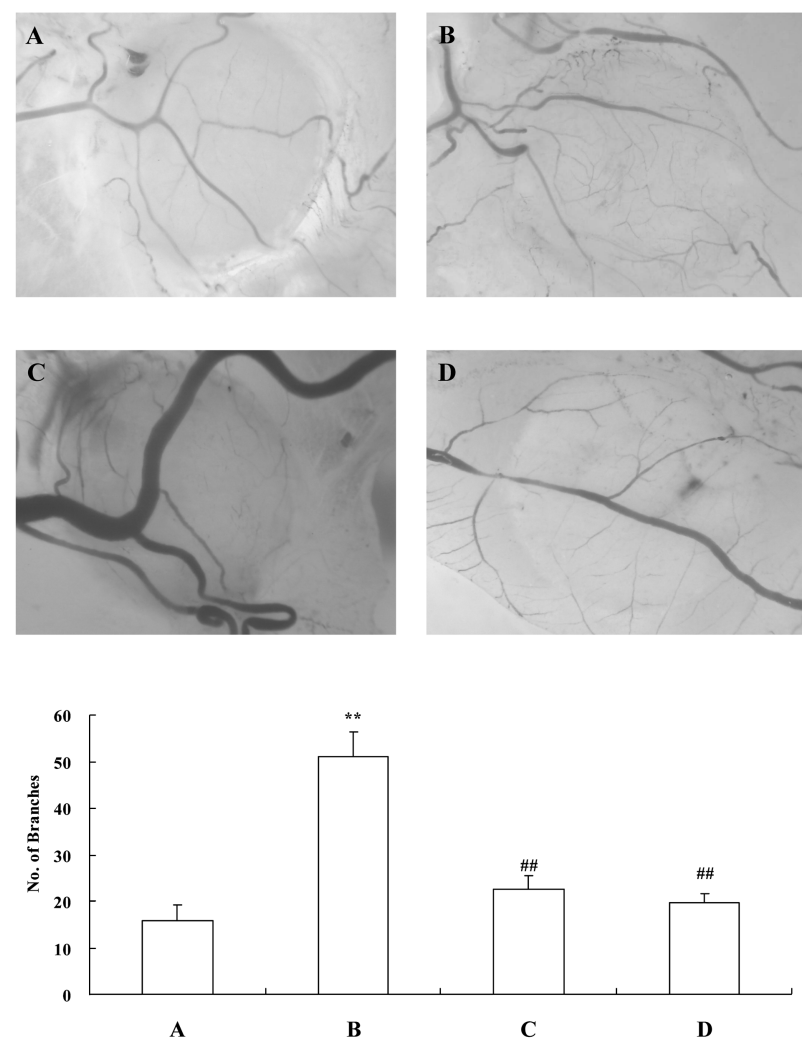

Fig. 3. FGb inhibits VEGF-induced angiogenesis in in the chick CAM assay. VEGF $(15 \mathrm{ng} / \mathrm{ml})$ or vehicle $(0.1 \%$ bovine serum albumin in phosphate buffered solution) was pipetted onto a cortisone dried filter disk placed on the CAM in an avascular area. After $30 \mathrm{~min}$ later, the disk was treated with various concentrations of FGb. Digital images (magnification: $\times 10$ ) of CAM sections exposed to filters were captured as representative images of vehicle-treated (A), VEGF-stimulated (B), VEGF with FGb (10 $\mu \mathrm{g} /$ disk)-treated (C), and FGb (100 $\mu \mathrm{g} /$ disk) (D). The bar graphs represent quantitation of new branches formed from existing blood vessels. $* * P<0.01$, compared to vehicle-treated control; ${ }^{\#} P<0.01$, compared to VEGF-treated group.

Panax ginseng C.A. Meyer, which has been used in traditional medicine in Korea and East Asian countries for more than 1000 years. Ginsenosides are triterpene saponins having a rigid steroidal skeleton with sugar moieties (Liu and Xiao, 1992). Up to now, more than 30 different ginsenosides have been isolated, each with a different set of properties. Within over 30 ginsenoside, $\operatorname{Rg}_{1}$, the most abundant and active component, has been reported to promote angiogenesis in vitro and in vivo (Sengupta et al., 2004; Liang et al., 2005), whereas $\operatorname{Rg}_{3}$ has been reported to exhibit in vitro and in vivo anti-carcinogenic, antimetastatic and anti-angiogenic effects (Mochizuki et al., 1995; Shinkai et al., 1996; Liu et al., 2000; Yun et al., 2001; Jin et al., 2006). In 
addition to the different biological effects of individual ginsenoside, recent studies have reported that orally administered ginsenosides are hardly decomposed by gastric juice or digestive enzymes (Karikura et al., 1991). The intact ginsenosides are poorly absorbed through small intestine. In contrast, their intestinal metabolites by colonic bacteria are absorbed and appear in the blood and urine (Hasegawa et al., 1996; Akao et al., 1998). One of the major metabolite known as compound $\mathrm{K}$ or M1, 20-O-( $\beta$-D-glucopyranosyl)-20(S)-protopanaxadiol, has been demonstrated to mediate in vivo anti-metastatic effects of ginsenosides (Hasegawa et al., 1997b). It is now well recognized that the deglycosylation process of ginsenoside by colonic bacteria is crucial for its pharmacological expression. Since intestinal bacteria are very changeable depending on host conditions, including diet, health, and even stress, bacterial ginsenoside-hydrolyzing potentials have been shown to differ among humans (Hasegawa et al., 1997b) and experimental mice (Hasegawa et al., 2000).

Since FGb is obtained by fermenting dried ginseng powder with bifidobacterium, it contains bioavailability-enhanced ginsenoside metabolite devoid of any disgusting odor. In addition, FGb is known to contain 20-O-( $\beta$-D-glucopyranosyl)-20(S)protopanaxadiol and 20(S)-protopanaxatriol, the biologically active compounds for anticancer effects (Korea patent 102004-0013653). Therefore, FGb can be used as an infallible remedy regardless of individual variations due to the condition of individual intestinal microflora. Our results further suggest that FGb is useful for the oral treatment of angiogenesis-related diseases such as cancer.

\section{ACKNOWLEDGMENTS}

This study was supported by Korea Research Foundation Grants funded by the Korean Government (MOEHRD, KRF2005-204-E00119 \& KRF-2006-005-J01102).

\section{REFERENCES}

Akao, T., Kida, H., Kanaoka, M., Hattori, M. and Kobashi, K. (1998). Intestinal bacterial hydrolysis is required for the appearance of compound $\mathrm{K}$ in rat plasma after oral administration of ginsenoside Rbl from Panax ginseng. J. Pharm. Pharmacol. 50, 1155-1160.

Blouw, B., Song, H., Tihan, T., Bosze, J., Ferrara, N., Gerber, H.P., Johnson, R.S. and Bergers, G. (2003). The hypoxic response of tumors is dependent on their microenvironment. Cancer Cell. 4, 133-146.

Folkman, J. (1971). Tumor angiogenesis: therapeutic implications. N. Engl. J. Med. 285, 1182-1186.
Hasegawa, H., Sung, J.H., Matsumiya, S., Uchiyama, M., Inoue, Y., Kasai, R. and Yamasaki, K. (1995). Reversal of daunomycin and vinblastin resistance in multidrug resistant P388 leukemia in vitro through enhanced cytotoxicity by triterpenoids. Planta Med. 61, 409-413.

Hasegawa, H., Sung, J.H., Matsumiya, S. and Uchiyama, M. (1996). Main ginseng saponin metabolites formed by intestinal bacteria. Planta Med. 62, 453-457.

Hasegawa, H., Sung, J.H. and Huh J.D. (1997a). Ginseng intestinal bacteria metabolite IH901 as a new antimetastatic agent. Arch. Pharm. Res. 20, 539-544.

Hasegawa, H., Sung, J.H. and Benno, Y. (1997b). Role of human intestinal Prevotella oris in hydrolyzing ginseng saponins. Planta Med. 63, 436-440.

Hasegawa, H., Lee, K.S., Nagaoka, T., Tezuka, Y., Uchiyama, M., Kadota, S. and Saiki, I. (2000). Pharmacokinetics of ginsenoside deglycosylated by intestinal bacteria and its transformation to biologically active fatty acid esters. Biol. Pharm. Bull. 23, 298-304.

Isner, J.M. and Asahara, T. (1999). Angiogenesis and vasculogenesis as therapeutic strategies for postnatal neovascularization. J. Clin. Invest. 103, 1231-1236.

Jin, J., Shahi, S., Kang, H.K., van Veen, H.W. and Fan, T.P. (2006). Metabolites of ginsenosides as novel BCRP inhibitors. Biochem. Biophys. Res. Commun. 345, 1308-1314.

Karikura, M., Miyase, T., Tanizawa, H., Taniyama, T. and Takino, Y. (1991). Studies on absorption, distribution, excretion and metabolism of ginseng saponins. VII. Comparison of the decomposition modes of ginsenoside- $R b_{1}$ and $-\mathrm{Rb}_{2}$ in the digestive tract of rats. Chem. Pharm. Bull. (Tokyo). 39, 23572361.

Leung, K.W., Pon, Y.L., Wong, R.N. and Wong, A.S. (2006). Ginsenoside-Rg1 induces vascular endothelial growth factor expression through the glucocorticoid receptor-related phosphatidylinositol 3-kinase/Akt and beta-catenin/T-cell factordependent pathway in human endothelial cells. J. Biol. Chem. 281, 36280-36288.

Liang, H.C., Chen, C.T., Chang, Y., Huang, Y.C., Chen, S.C. and Sung, H.W. (2005). Loading of a novel angiogenic agent, ginsenoside $\mathrm{Rg}_{1}$ in an acellular biological tissue for tissue regeneration. Tissue Eng. 11, 835-846.

Lin, C.M., Chang, H., Chen, Y.H., Li, S.Y., Wu, I.H. and Chiu, J.H. (2006). Protective role of wogonin against lipopolysaccharide-induced angiogenesis via VEGFR-2, not VEGFR-1. Int. Immunopharmacol. 6, 1690-1698.

Liu, C.X. and Xiao, P.G., (1992). Recent advances on ginseng research in China. J. Ethnopharmacol. 36, 27-38.

Liu, W.K., Xu, S.X. and Che, C.T. (2000). Anti-proliferative effect of ginseng saponins on human prostate cancer cell line. Life Sci. 67, 1297-1306.

Mochizuki, M., Yoo, Y.C., Matsuzawa, K., Sato, K., Saiki, I., Tono-oka, S., Samukawa, K. and Azuma, I. (1995). Inhibitory effect of tumor metastasis in mice by saponins, ginsenoside$\mathrm{Rb}_{2}, 20(\mathrm{R})$ - and $20(\mathrm{~S})$-ginsenoside- $\mathrm{Rg}_{3}$, of red ginseng. Biol. Pharm. Bull. 18, 1197-1202.

Mousa, S.A., Mohamed, S., Wexler, E.J. and Kerr, J.S. (2005). Antiangiogenesis and anticancer efficacy of TA138, a novel alphavbeta3 antagonist. Anticancer Res. 25, 197-206.

Rosenblatt, M.I. and Azar, D.T. (2006). Anti-angiogenic therapy: Prospects for treatment of ocular tumors. Semin. Ophthalmol. 21, 151-160.

Sengupta, S., Toh, S.A., Sellers, L.A., Skepper, J.N., Koolwijk, P., Leung, H.W., Yeung, H.W., Wong, R.N., Sasisekharan, R. and 
Fan, T.P. (2004). Modulating angiogenesis: the yin and the yang in ginseng. Circulation. 110, 1219-1225.

Shin, H.R., Kim, J.Y., Yun, T.K., Morgan, G. and Vainio, H. (2000). The cancer-preventive potential of Panax ginseng: a review of human and experimental evidence. Cancer Causes Control. 11, 565-576.

Shinkai, K., Akedo, H., Mukai, M., Imamura, F., Isoai, A., Kobayashi, M. and Kitagawa, I. (1996). Inhibition of in vitro tumor cell invasion by ginsenoside $\mathrm{Rg}_{3}$. Jpn. J. Cancer Res. 87, 357362.

Tanizawa, H., Karikuma, M., Miyase, T. and Takino, Y. (1993). Studies on the metabolism and/or decomposition and distribution of ginsenoside $\mathrm{Rb}_{2}$ in rats. Proc. 6th Int. Ginseng Symp.
Seoul. p.187-194.

Xu, Q.F., Fang, X.L. and Chen, D.F. (2003). Pharmacokinetics and bioavailability of ginsenoside $\mathrm{Rb}_{1}$ and $\mathrm{Rg}_{1}$ from Panax notoginseng in rats. J. Ethnopharmacol. 84, 187-192.

Yue, P.Y., Wong, D.Y., Wu, P.K., Leung, P.Y., Mak, N.K., Yeung, H.W., Liu, L., Cai, Z., Jiang, Z.H., Fan, T.P. and Wong, R.N. (2006). The angiosuppressive effects of 20(R)-ginsenoside $\mathrm{Rg}_{3}$. Biochem. Pharmacol. 72, 437-445.

Yun, T.K., Lee, Y.S., Lee, Y.H., Kim, S.I. and Yun, H.Y. (2001). Anticarcinogenic effect of Panax ginseng C.A. Meyer and identification of active compounds. J. Korean Med. Sci. 16, S6-18. 